patients was stratified into two cohorts: those who had received ADT for longer than the median of 697 days ('long-term' group) and those receiving therapy for a shorter period.

Long-term ADT was associated with an increased risk of osteopenia/osteoporosis and of nonpathologic fractures, although there was no significant increase in the likelihood of pathologic fractures. Men over 85 years old were more likely to suffer pathologic or nonpathologic fractures than their younger counterparts, whereas African-American race appeared to be a protective factor. Existing bone metastasis, a common comorbidity, was a risk factor for pathologic fracture only.

The authors conclude that patients are at risk of bone complications both from ADT and from the disease itself. They suggest that further profiling of men at greatest risk will support their management.

Original article Krupski TL et al. (2004) Natural history of bone complications in men with prostate carcinoma initiating androgen deprivation therapy. Cancer 101: 541-549

\section{Towards tailored chemotherapy for NSCLC}

An individual's response to chemotherapy may be influenced by single nucleotide polymorphisms (SNPs) at a variety of loci. Isla and colleagues from the Spanish Lung Cancer Group have considered whether SNPs in four relevant genes (ERCC1, XPD, RRM1 and MDR1) predict survival in non-small-cell lung cancer (NSCLC) patients treated with docetaxel-cisplatin.

Using genomic DNA from 62 patients with advanced NSCLC, the investigators carried out a one-step assay, to amplify the regions containing the SNPs and to generate fluorescent signals indicating which alleles were present. They then analyzed the patients' survival, time to progression and response to therapy according to SNP genotype.

Patients with the wild-type $(\mathrm{C} / \mathrm{C})$ genotype at ERCC1 codon 118 showed significantly better survival, and longer time to progression, than those bearing one or two copies of the $\mathrm{T}$ allele at this position. No statistically significant differences were shown in response to therapy. The XPD, RRM1 and MDR1 SNPs tested showed no significant association with survival, time to progression or response, although this may have been due to the small number of patients studied.

The authors suggest that the predictive value of the ERCC1 118 genotype-if validated in an ongoing, larger study-might prove valuable in the design of tailored chemotherapy trials. Survival of patients bearing the $\mathrm{T}$ allele might be improved using non-cisplatin combinations.

Original article Isla D et al. (2004) Single nucleotide polymorphisms and outcome in docetaxel-cisplatin-treated advanced non-small-cell lung cancer. Ann Oncol 15: 1194-1203

\section{Statin therapy: long-term mortality and cancer incidence}

The Scandinavian Simvastatin Survival Study (4S), which ended in 1994, demonstrated that cholesterol-lowering with simvastatin reduced the rates of coronary events and all-cause mortality. Following the trial, which included a median follow-up period of 5.4 years, most patients received open-label lipid-lowering treatment. Strandberg et al. now report on their 5-year extended follow-up, which studied causespecific mortality and cancer incidence after the end of the double-blind follow-up period.

The authors compared records of cause-ofdeath and cancer incidence with the 4S patient register. Mortality was significantly lower during the median 10.4-year follow-up in the 414 patients originally allocated to the simvastatin group than in the 468 patients in the placebo group (relative risk 0.85 [95\% confidence interval (Cl) 0.74-0.97]; $P=0.02)$. Most of this difference was due to a lower rate of coronary mortality in the simvastatin group. There had been fewer cancer deaths in the simvastatin group compared with placebo after extended follow-up, although this difference was not statistically significant ( 85 vs 100 ; relative risk 0.81 [95\% Cl 0.60-1.08]; $P=0.14$ ). Excluding cancers in the same categories as those diagnosed before randomization, cancer incidence was slightly, although not significantly, lower in the simvastatin group compared with placebo (227 vs 248; relative risk 0.88 [95\% $\mathrm{Cl} 0.73-1.05]$; $P=0.147$ ). Further, simvastatin treatment was not associated with an increased incidence of any particular type of cancer.

Original article Strandberg TE et al. (2004) Mortality and incidence of cancer during 10-year follow-up of the Scandinavian Simvastatin Survival Study (4S). Lancet 364: 771-777 